\title{
Pharmacometabolic response to pirfenidone in pulmonary fibrosis detected by MALDI-FTICR-MSI
}

\author{
Na Sun ${ }^{1,6}$, Isis E. Fernandez ${ }^{2,6}$, Mian Wei $^{1}$, Michael Witting ${ }^{3}$, Michaela Aichler $^{1}$, \\ Annette Feuchtinger ${ }^{1}$, Gerald Burgstaller ${ }^{2}$, Stijn E. Verleden ${ }^{4}$, \\ Philippe Schmitt-Kopplin ${ }^{3}$, Oliver Eickelberg ${ }^{2,5,7}$ and Axel Walch ${ }^{1,7}$
}

Affiliations: ${ }^{1}$ Research Unit Analytical Pathology, Helmholtz Zentrum München, German Research Center for Environmental Health $(\mathrm{GmbH})$, Neuherberg, Germany. ${ }^{2}$ Comprehensive Pneumology Center, Helmholtz Zentrum München, Ludwig Maximilian University München, Member of the German Center for Lung Research (DZL), Munich, Germany. ${ }^{3}$ Research Unit Analytical BioGeoChemistry, Helmholtz Zentrum München, German Research Center for Environmental Health $(\mathrm{GmbH})$, Neuherberg, Germany. ${ }^{4}$ Laboratory of Pneumology, Dept of Chronic Diseases, Metabolism and Aging, KU Leuven, Leuven, Belgium. ${ }^{5}$ Division of Respiratory Sciences and Critical Care Medicine, Dept of Medicine, University of Colorado, Denver, CO, USA.

${ }^{6}$ These authors contributed equally to this work. ${ }^{7}$ These authors contributed equally to this work.

Correspondence: Axel Walch, Research Unit Analytical Pathology, Helmholtz Zentrum München, German Research Center for Environmental Health $(\mathrm{GmbH})$, Ingolstädter Landstrasse 1, 85764 Neuherberg, Germany. E-mail: axel.walchahelmholtz-muenchen.de

@ERSpublications

MALDI-FTICR-mass spectrometry imaging detects the pharmacometabolic effect of pirfenidone in fibrosis http://ow.ly/zKee30l1tNs

Cite this article as: Sun N, Fernandez IE, Wei M, et al. Pharmacometabolic response to pirfenidone in pulmonary fibrosis detected by MALDI-FTICR-MSI. Eur Respir J 2018; 52: 1702314 [https://doi.org/ 10.1183/13993003.02314-2017].

ABSTRACT Idiopathic pulmonary fibrosis (IPF) is a fatal condition that reduces life expectancy and shows a limited response to available therapies. Pirfenidone has been approved for treatment of IPF, but little is known about the distinct metabolic changes that occur in the lung upon pirfenidone administration.

Here, we performed a proof-of-concept study using high-resolution quantitative matrix-assisted laser desorption/ionisation Fourier-transform ion cyclotron resonance mass spectrometry imaging (MALDIFTICR-MSI) to simultaneously detect, visualise and quantify in situ endogenous and exogenous metabolites in lungs of mice subjected to experimental fibrosis and human patients with IPF, and to assess the effect of pirfenidone treatment on metabolite levels.

Metabolic pathway analysis and endogenous metabolite quantification revealed that pirfenidone treatment restores redox imbalance and glycolysis in IPF tissues, and downregulates ascorbate and aldarate metabolism, thereby likely contributing to in situ modulation of collagen processing. As such, we detected specific alterations in metabolite pathways in fibrosis and, importantly, metabolic recalibration following pirfenidone treatment.

Together, these results highlight the suitability of high-resolution MALDI-FTICR-MSI for deciphering the therapeutic effects of pirfenidone and provide a preliminary analysis of the metabolic changes that occur during pirfenidone treatment in vivo. These data may therefore contribute to improvement of currently available therapies for IPF.

This article has supplementary material available from erj.ersjournals.com

Received: Nov 092017 | Accepted after revision: July 152018

Copyright OERS 2018 


\section{Introduction}

Idiopathic pulmonary fibrosis (IPF) is a chronic progressive and diffuse parenchymal lung disease that leads to loss of organ function. IPF is characterised by replacement of lung parenchyma with fibrotic tissue, aberrant fibroblast-myofibroblast activation, epithelial hyperplasia and injury, and immune dysregulation [1]. IPF is considered to be an incurable lethal disease, with median survival ranging from 2 to 3 years, worse than many cancers.

Pirfenidone (5-methyl-1-phenyl-2-(1H)-pyridone) is an orally active pyridine molecule that exhibits antifibrotic and anti-inflammatory activities $[2,3]$. It is approved for IPF treatment worldwide, including the European Union and the USA, where it is one of two drugs approved for use in IPF patients [4]. Although pirfenidone is a first-line drug for IPF treatment [5], its precise mechanism of action has not been elucidated to date [6]. Here, in an effort to better guide treatment of IPF, we seek to better understand the detailed mechanism of pirfenidone activity.

In situ metabolite imaging is a high-throughput, nontargeted metabolomic technique that has been refined by ultra-sensitive and high-resolution quantitative matrix-assisted laser desorption/ionisation mass spectrometry imaging (MALDI-MSI), with Fourier-transform ion cyclotron resonance (FTICR) and Orbitrap [7-11]. High-mass-resolution MALDI-MSI enables simultaneous mapping of spatial distribution for a wide range of low-molecular-weight metabolites, including central metabolic pathway metabolites, redox-related metabolites, nucleotide derivatives, lipids and amino acids, directly within morphologically intact tissue sections. MALDI-MSI mass spectra are acquired by measuring spots in a predefined raster across a tissue section, resulting in a two-dimensional distribution map for each measured molecule [12, 13]. Therefore, MALDI-MSI allows acquisition of cellular expression profiles, while preserving cellular and molecular integrity. This technology excels at imaging the spatial organisation of molecules within histological features [14] and can characterise molecular interaction networks of several functionally distinct pathways [15].

Metabolic changes in IPF pathogenesis, particularly patient-specific responses to drug treatment, remain poorly understood. Recent liquid-based metabolomic studies of IPF have reported several disrupted pathways, including sphingolipid metabolism, glycolysis, the tricarboxylic acid (TCA) cycle, arginine metabolism, ATP, glutathione biosynthesis and ornithine aminotransferase $[16,17]$. We recently reported that high-resolution MALDI-FTICR-MSI can accurately detect the distribution and pharmacokinetics of pirfenidone and pirfenidone-related metabolites (5-hydroxymethyl pirfenidone and 5-carboxy pirfenidone) in healthy mouse tissues. Notably, in that study, we did not observe consistent effects of pirfenidone, or its metabolites, on known metabolic pathways [18].

Here, to explore endogenous metabolite dynamics upon pirfenidone treatment in fibrotic lungs, we used high-resolution MALDI-FTICR-MSI and performed the first tissue-based in situ metabolite imaging on treatment-naive and pirfenidone-treated IPF patients, as well as an experimental mouse model of this disease. We provide a proof-of-concept for the use of this technique to assess dynamic metabolic changes occurring in IPF and in patients treated with pirfenidone.

\section{Materials and methods Experimental mouse model of pulmonary fibrosis}

All animal experiments utilised 8-10-week-old female C57BL/6N mice (Charles River Laboratories, Wilmington, MA, USA) and were performed in accordance with recommendations in the Guide for the Care and Use of Laboratory Animals of the National Institutes of Health. Pulmonary fibrosis in mice was established as described (ethical approval TVA21-12) [19]. Briefly, bleomycin (3 U. $\mathrm{kg}^{-1}$ ) or PBS was applied intratracheally as a single dose. From day 7 to 14 post-treatment, a subset of both bleomycin-treated and control animals was administered oral pirfenidone daily (Selleckchem, Houston, TX, USA) (supplementary material). All animals (PBS $n=3$, PBS+pirfenidone $n=3$, bleomycin $n=3$ and bleomycin+pirfenidone $n=3$ ) were sacrificed on day 14 .

\section{Human lung samples}

This study was approved by the hospital ethics and university biosafety committees in Leuven, Belgium. Explanted lung tissue from IPF patients that were either untreated or under pirfenidone treatment, or donor controls, were collected at KU Leuven (ethical approval ML6385). Protocols for processing and sampling lung specimens have been described in detail [20]. Briefly, each lung was inflated with air to a transpulmonary pressure of $30 \mathrm{cmH}_{2} \mathrm{O}$, then deflated to $10 \mathrm{cmH}_{2} \mathrm{O}$ while freezing in liquid nitrogen vapour; frozen samples were stored at $-80^{\circ} \mathrm{C}$. Lungs (IPF $n=4$, pirfenidone-treated IPF $n=3$ and donor $\mathrm{n}=4$ ) were cut into slices $2 \mathrm{~cm}$ thick; cores with a diameter of $1.4 \mathrm{~cm}$ were excised and processed as described previously [21]. 


\section{Immunofluorescence in frozen tissue}

Slides containing sections of frozen lung were fixed by immersion in $2 \%$ paraformaldehyde for 5 min, then dipped in distilled water, followed by a short wash in Tris-buffered saline with Tween 20 (TBS-T). These were blocked by immersion in 3\% hydrogen peroxide/distilled water solution (10 min), followed by a 3\% bovine serum albumin (BSA)/TBS-T buffer solution $(20 \mathrm{~min})$, to prevent nonspecific binding. Slides were stained with collagen type 1 (COL1) antibody (Rockland Immunochemicals, Pottstown, PA, USA), diluted (1:250) with antibody diluent (Zytomed Systems, Berlin, Germany). COL1 antibody staining was performed in a wet chamber at $4^{\circ} \mathrm{C}$, overnight. Slides were then rinsed three times with TBS-T and a 1:250 dilution of secondary antibody (Alexa Fluor 568 donkey anti-rabbit; Invitrogen, Carlsbad, CA, USA) was applied, followed by incubation at room temperature $(1 \mathrm{~h})$ in the dark. Slides were counterstained with 4',6-diamidino-2-phenylindole (1:2500; Sigma-Aldrich, St Louis, MO, USA) for $1 \mathrm{~min}$ and then rinsed three times with TBS-T buffer. They were then covered with Fluorescence Mounting Medium (Dako, Hamburg, Germany) and scanned using an AxioScan.Z1 digital slide scanner (Zeiss, Goettingen, Germany), equipped with a $\times 20$ magnification objective.

\section{MALDI-FTICR-MSI}

For the endogenous metabolomics study, MALDI-FTICR-MSI (solariX 7T; Bruker Daltonics, Billerica, MA, USA) measurements were performed in the mass range of $\mathrm{m} / \mathrm{z} 50-1000$ in negative ion mode $(\mathrm{MH}-)$ at a frequency of $500 \mathrm{~Hz}$, using 50 laser shots and a spatial resolution of $70 \mu \mathrm{m}$.

For the drug imaging study of pirfenidone and its related metabolites, 5-hydroxymethyl pirfenidone and 5-carboxy pirfenidone, MALDI-FTICR-MSI (solariX 7T) was performed in continuous accumulation of selected ions (CASI) mode. The mass-selective quadrupole was set to $186.1 \mathrm{~m} / z$, with a window of 130 $\mathrm{m} / \mathrm{z}$. Mass spectra were acquired with a $70 \mu \mathrm{m}$ spatial resolution in positive mode, using 100 laser shots, at a frequency of $1 \mathrm{kHz}$.

\section{Metabolite identification by liquid chromatography-MS and tissue MS/MS, and pathway analysis} Targeted metabolite identification by liquid chromatography (LC)-MS was performed as described [22]. Complementary experiments for metabolite identification using tissue tandem MS/MS analysis were performed with MALDI-FTICR-MSI (solariX 7T) using CASI mode, which allows target ion selection in quadrupole, and fragmented using collision-induced dissociation. Metabolites were identified or estimated by comparing MS/MS spectra with standard compounds or by matching accurate mass with databases, as described elsewhere [15, 22, 23]: METLIN (http://metlin.scripps.edu), MassTRIX (http:// masstrix3.helmholtz-muenchen.de/masstrix3), METASPACE (http://annotate.metaspace2020.eu) [24] and the Human Metabolome Database (www.hmdb.ca) [25]. These databases were used in conjunction with MetaboAnalyst (www.metaboanalyst.ca)[26] and KEGG (www.genome.jp/kegg) for pathway analysis. Details of MALDI-MSI tissue preparation, data processing and statistical analysis, relative quantification of endogenous metabolites, and metabolite identification by LC-MS are provided in the supplementary material.

\section{Results}

Detection of pirfenidone and endogenous metabolites in situ in experimental fibrosis

To explore the distribution and effects of pirfenidone in fibrotic lung tissue, we induced experimental pulmonary fibrosis in mice using bleomycin. Pirfenidone $\left(500 \mathrm{mg} \cdot \mathrm{kg}^{-1}\right.$ ) was administered once per day by oral gavage from day 7 to 14 , based on the previously determined time taken to reach the maximum concentration $\left(t_{\max }\right)$ in normal mouse tissues [18]. On day 14, mice were sacrificed $45 \mathrm{~min}$ after final pirfenidone administration and lung tissue was harvested. Histological analysis of bleomycin-treated samples revealed destruction of normal tissue architecture, excessive accumulation of extracellular matrix (ECM) and decreased lung compliance compared with PBS-treated controls (figure $1 \mathrm{a}$ and b). In bleomycin +pirfenidone-treated mice, we observed less pronounced lung fibrosis and significantly improved lung function, as demonstrated by improved compliance compared with that observed in bleomycin-treated animals (figure 1a and b). The pirfenidone metabolites 5-hydroxymethyl pirfenidone $(\mathrm{m} / z 202.0863)$ and 5-carboxy pirfenidone $(\mathrm{m} / \mathrm{z}$ 216.0655) were globally more abundant in lung tissue from bleomycin +pirfenidone-treated mice than unaffected controls treated with pirfenidone (figure 1c). Notably, we observed increased pirfenidone accumulation in conserved areas of fibrotic lungs from bleomycin+pirfenidone-treated mice, corresponding to regions with lower COL1 content within fibrotic areas (figure 1f).

To identify alterations in endogenous metabolites, we performed in situ imaging of major members of key metabolic pathways in fibrotic lungs from bleomycin+pirfenidone-treated mice. We identified nucleotide derivatives, as well as central metabolic pathway and redox-related metabolites. For some (e.g. GMP, UMP and glucose 6-phosphate), their distribution patterns correlated with fibrotic areas across micro-regions (figure $1 \mathrm{~d}$ and e). We then performed unsupervised hierarchical clustering $[27,28]$ of metabolites in lungs 
a)
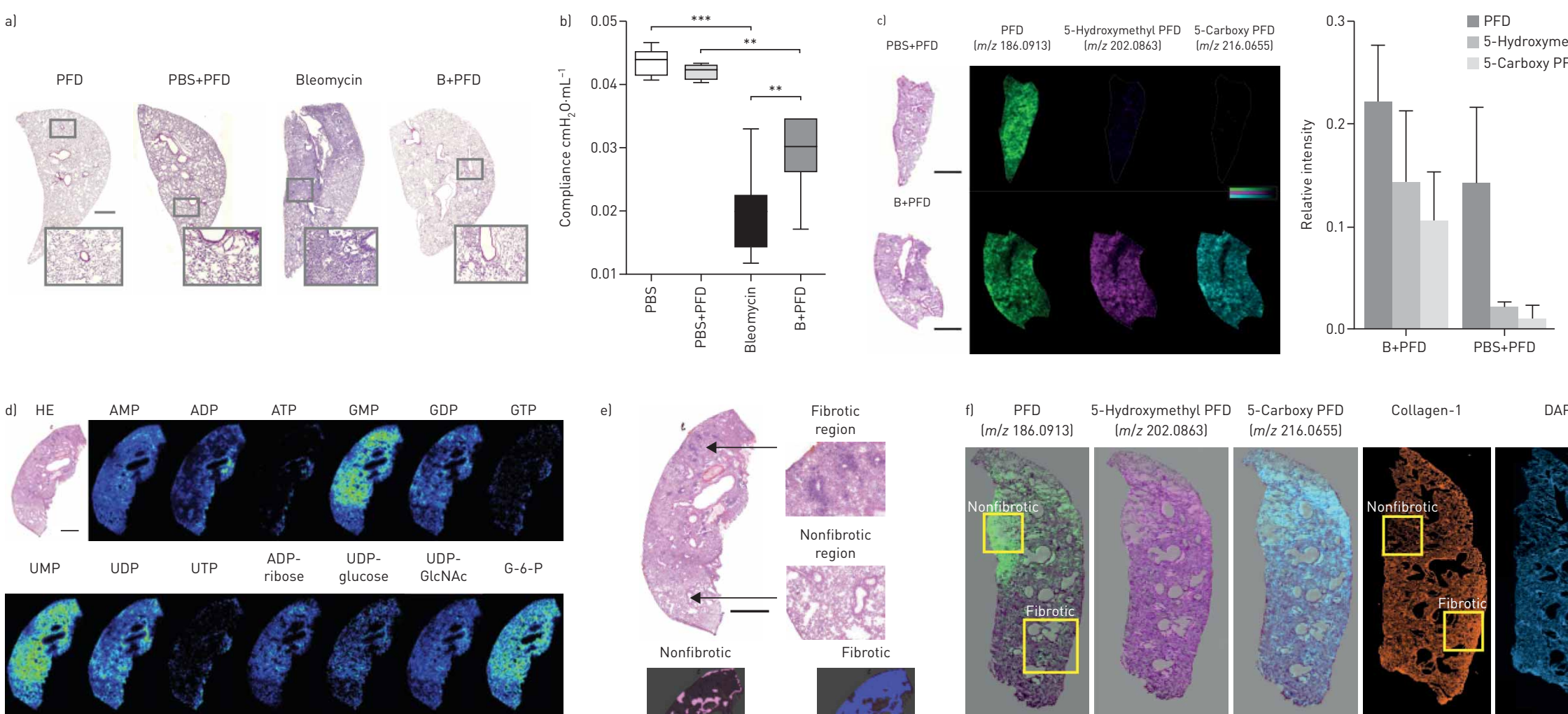

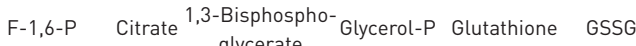
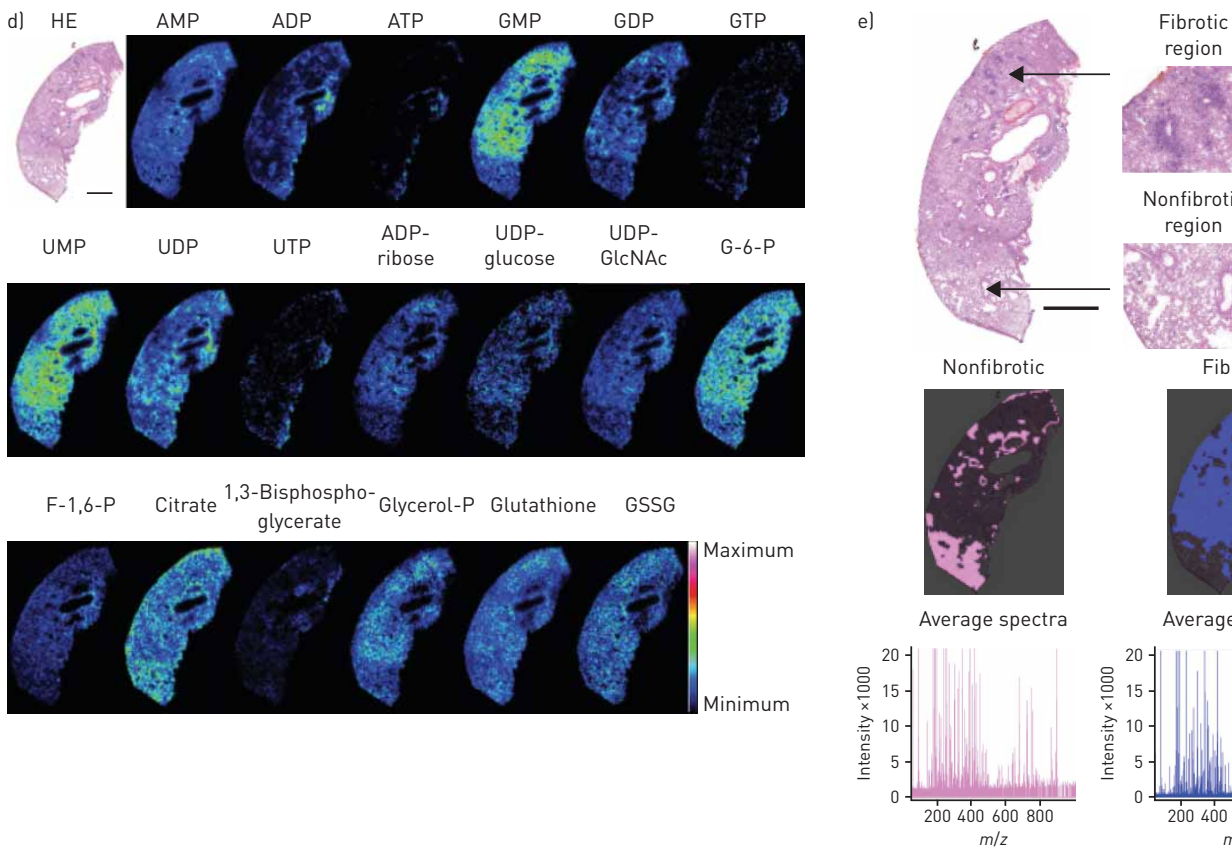

region

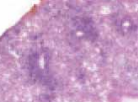

Nonfibrotic region
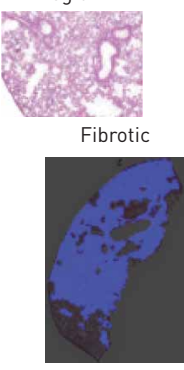

Average spectra

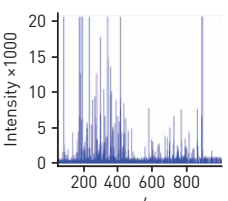

$\mathrm{m} / \mathrm{z}$

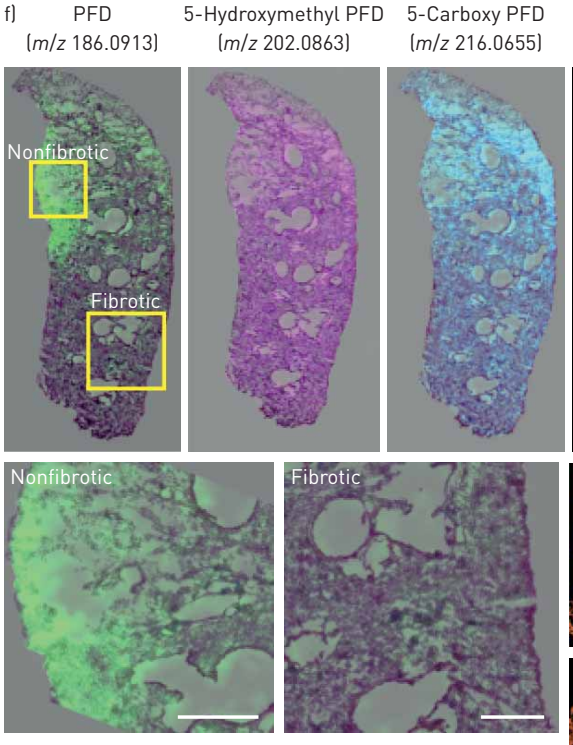

Collagen-1

DAPI

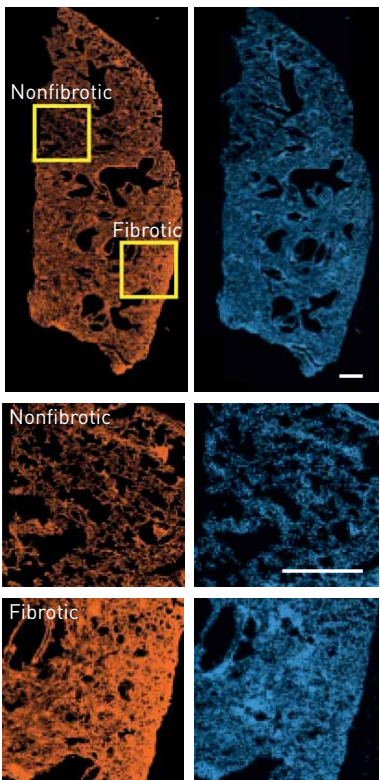

FIGURE 1 Pirfenidone (PFD) detection in bleomycin-induced pulmonary fibrosis mouse lungs. a) HE staining of sectioned lungs from mice treated with PBS, PBS+PFD, bleomycin to induce fibrosis and B+PFD. Scale bar: $1000 \mu \mathrm{m}$. b) Lung compliance measurements of mice from each treatment group. Data are shown as mean \pm SD. ${ }^{* *}$ : $p \leqslant 0.01$; $* * *$ : $p \leqslant 0.001$. c) Left: mass spectrometry imaging distribution of PFD and its related metabolites, 5-hydroxymethyl PFD and 5-carboxy PFD, in lungs from PBS+PFD- and B+PFD-treated mice. Scale bars: $1000 \mu$ m. Right: relative quantification of PFD and related metabolites in lungs from PBS+PFD- and B+PFD-treated mice. d) Heterogeneous distribution pattern of central pathway-related endogenous metabolites in lung tissue from B+PFD-treated mice. Scale bar: $1000 \mu \mathrm{m}$. e) HE images with zoomed regions of nonfibrotic and fibrotic tissue. Spatial segmentation of histological components through unsupervised hierarchical clustering reflects the histological components of fibrotic (blue) and nonfibrotic (pink) areas. Scale bar: $1000 \mu \mathrm{m}$. f) Immunofluorescence staining for collagen type 1 in sectioned lungs from the B+PFD-treated group. Scale bars: $500 \mu \mathrm{m}$. B+PFD: bleomycin+PFD; HE: haematoxylin and eosin; GlcNAc: N-acetylglucosamine; G-6-P: glucose 6-phosphate; F-1,6-P: fructose 1,6-bisphosphate; glycerol-P: glycerol monophosphate; GSSG: glutathione disulfide; DAPI: 4',6-diamidino-2-phenylindole. 
from bleomycin+pirfenidone-treated animals, and generated a segmentation map of fibrotic and nonfibrotic areas based on different MSI spectrum clusters (figure 1e). These data were in agreement with microscopic inspection conducted by histopathologists.

MALDI-FTICR-MSI analysis of glycolysis and the TCA cycle pathways after pirfenidone treatment We next compared expression of several metabolites from glycolysis and the TCA cycle, two central metabolic pathways, in lungs from animals in all treatment groups (figure 2). We observed that glucose 6-phosphate was increased during fibrosis in bleomycin-treated mice, whereas 1,3-bisphosphoglycerate was decreased in lungs from bleomycin- and bleomycin+pirfenidone-treated animals; fructose 1,6-bisphosphate levels were unchanged. When pyruvate, which derives from 1,3-bisphosphoglycerate, enters the hexosamine biosynthesis pathway, it is transformed into UDP- $N$-acetylglucosamine.
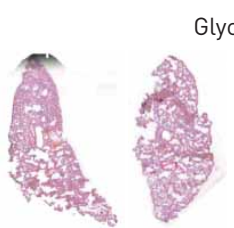

PBS
Glycolysis

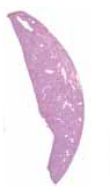

PBS+PFD Bleomycin

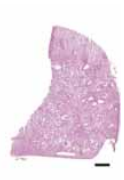

$B+P F D$
Glucose

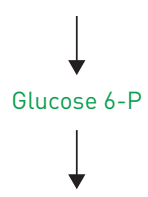

Fructose 6-P

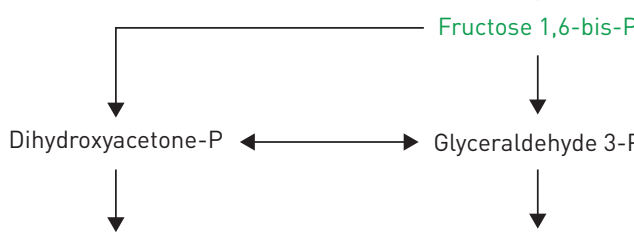

Glycerol-P

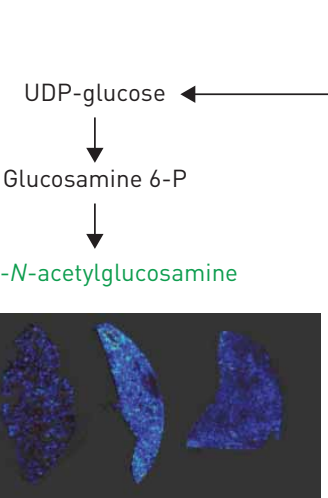

PBS

PBS+PFD Bleomycin B+PFD
1,3-Bisphosphoglycerate Pyruvate

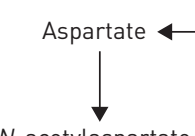

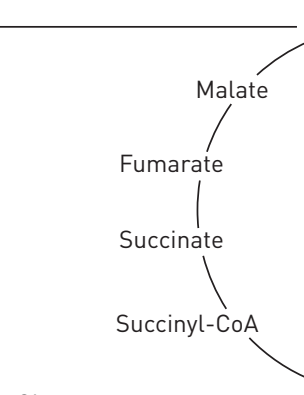

Glutamate
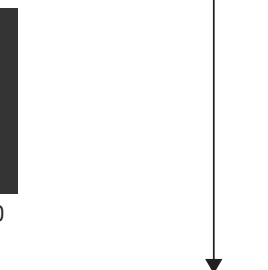

Oxaloacetate

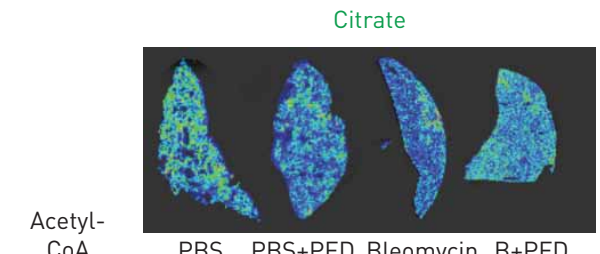
CoA<smiles>CC(C(=O)O)C(C)(C)C</smiles>

PBS PBS+PFD Bleomycin B+PFD

FIGURE 2 Analysis of glycolysis and tricarboxylic acid (TCA) cycle metabolism by high-resolution quantitative matrix-assisted laser desorption/ionisation Fourier-transform ion cyclotron resonance mass spectrometry imaging in fibrotic mice. Haematoxylin and eosin sections of lungs treated with PBS, PBS+PFD, bleomycin and B+PFD are shown in the top left. Scale bar: $1000 \mu \mathrm{m}$. Representative metabolite images of selected steps (in green) of glycolysis and the TCA cycle are shown. PFD: pirfenidone; B+PFD: bleomycin+PFD; P: phosphate; CoA: coenzyme A. 
UDP- $N$-acetylglucosamine participates in hyaluronan synthesis [29], an ECM component that is highly produced by myofibroblasts in bleomycin-induced fibrosis and IPF [30]. Here, we found significant enhancement of UDP- $N$-acetylglucosamine in fibrotic lungs from bleomycin-treated mice. However, unlike UDP- $N$-acetylglucosamine, citrate levels were similar in all groups. These data provide preliminary evidence for metabolic changes occurring in lungs during bleomycin-induced fibrosis and pirfenidone treatment in mice

\section{Metabolic effects of pirfenidone treatment in bleomycin-induced lung fibrosis}

We next performed metabolic pathway enrichment analysis, comparing PBS controls and bleomycin-induced fibrosis (figure 3a). Control regions were chosen from PBS-treated mice by selecting healthy, noncollapsed areas from each lung section. Fibrotic regions were selected from bleomycin-treated mice, based on histological features and the fibrotic clusters identified by spatial segmentation (figure 1e). In total, 1402 molecules were found to be significantly different between control and fibrotic tissue, with 409 molecules enriched and 993 molecules reduced in fibrotic regions. Furthermore, 536 of these were annotated in the METLIN database; these were putatively assigned to 57 lipids (24 reduced and 33 enriched in fibrotic regions), 234 peptides (223 reduced and 11 enriched in fibrotic regions) and 245 other metabolites. Metabolites responsible for metabolic differentiation were then subjected to pathway enrichment analysis (figure 3 and table 1). Regulated metabolites driving pathway significance are listed in supplementary table $\mathrm{S} 1$. We found glycolysis/gluconeogenesis metabolism was significantly reduced in pulmonary fibrosis, whereas ascorbate and aldarate metabolism, amino sugar and nucleotide sugar metabolism, the pentose phosphate pathway, pentose and glucuronate interconversions, and fructose and mannose metabolism were significantly enhanced in bleomycin-induced fibrosis (figure 3a, table 1 and supplementary table S1).

To elucidate the pharmacometabolic effects of pirfenidone, a second comparison was performed on fibrotic animals treated with or without a 7-day oral dose of pirfenidone (figure 3b). From this analysis, 115 discriminative $\mathrm{m} / \mathrm{z}$ species were identified. After database search and pathway analysis, ascorbate and aldarate metabolism, which were enhanced in bleomycin-induced fibrosis, were found to be downregulated after pirfenidone treatment (figure 3b, table 1 and supplementary table S1). This suggests a vital role for pirfenidone in switching systematic metabolite profiles in disease. Ascorbate contributes to efficient hydroxylation of hydroxyproline in elastin, collagen and other ECM proteins with collagenous domains, thus supporting an important role of pirfenidone in modulating de novo ECM production in bleomycin-induced fibrosis at the metabolic level.

To further clarify the role of pirfenidone during fibrosis, we performed metabolic pathway analysis on healthy mice treated with pirfenidone (PBS+pirfenidone). These data revealed high metabolic similarity between the PBS+pirfenidone group and mice treated with PBS alone (figures 2, 3d and 4). This is consistent with our previous pharmacokinetic study of pirfenidone in healthy mouse tissues, in which we did not observe significant effects of pirfenidone, or its metabolites, on known metabolic pathways [18]. We then compared lungs from fibrotic animals treated with bleomycin+pirfenidone and healthy mice treated with pirfenidone (PBS+pirfenidone). In total, 1076 molecules were significantly different between these groups, with 427 molecules enriched and 649 molecules reduced in fibrotic animals treated with pirfenidone. Pathway enrichment analysis identified multiple discriminative pathways, including glycolysis/ gluconeogenesis, amino sugar and nucleotide sugar metabolism, the pentose phosphate pathway, pentose and glucuronate interconversions, and fructose and mannose metabolism (figure 3c). Notably, ascorbate and aldarate metabolism remained unchanged in fibrotic animals treated with pirfenidone compared with controls (PBS+pirfenidone). These results further support the effects of pirfenidone treatment on ascorbate and aldarate metabolism.

Next, we performed in situ detection of key metabolites from the major metabolic pathways reported earlier. We selected eight representative significantly regulated metabolites (four up, four down) that were strongly differentially regulated in fibrosis (figure $3 \mathrm{~d}$ and e). Among the downregulated metabolites in fibrosis, we included phosphoenol pyruvate, bisphosphoglycerate, phosphatidic acid and phosphatidylethanolamine. Interestingly, phosphatidic acid and phosphatidylethanolamine were dramatically depleted in fibrosis, and these were partially restored by pirfenidone treatment. Conversely, ascorbic acid, glucose 6-phosphate, $\mathrm{N}$-acetylglucosamine phosphate and UDP- $N$-acetyl-D-galactosamine were upregulated during fibrosis. Remarkably, ascorbic acid was significantly decreased with pirfenidone treatment, supporting our pathway analysis.

\section{Quantification of central pathway-related endogenous metabolites in healthy and fibrotic lung}

To further investigate metabolite dynamics in disease conditions, we compared the relative abundance of 25 endogenous metabolites (nucleotide derivatives, central pathway metabolites, prostaglandins and 

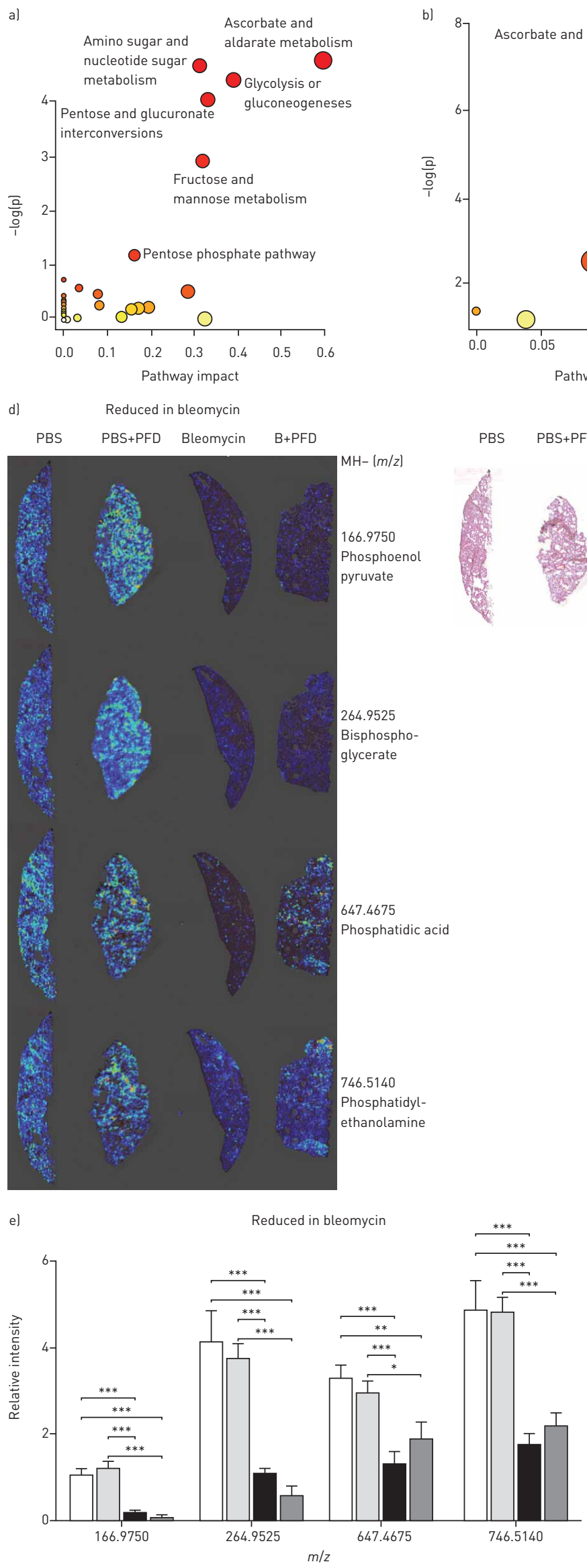
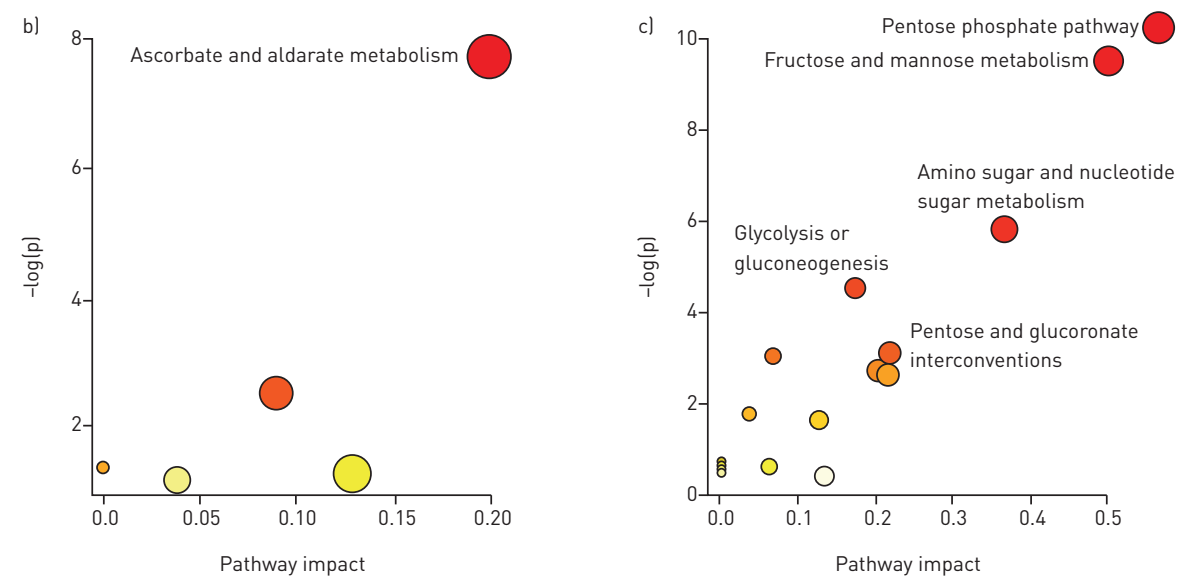

Enhanced in bleomycin
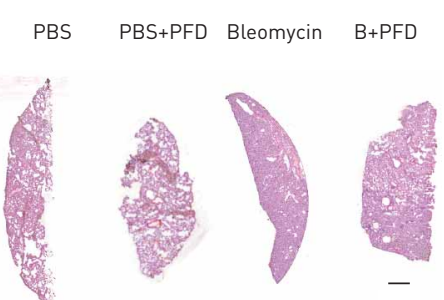

PBS+PFD Bleomycin B+PFD

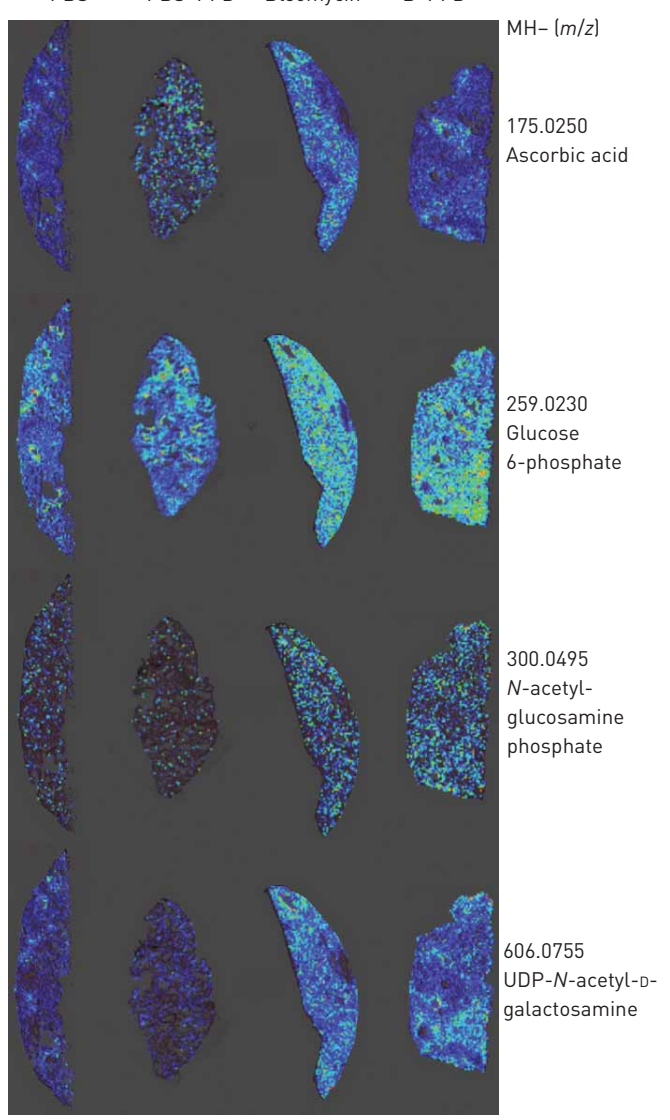

Enhanced in bleomycin

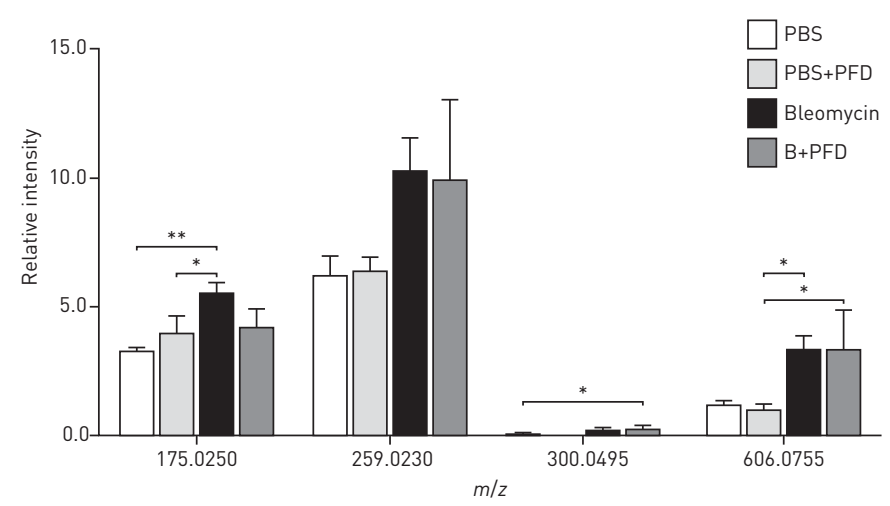


FIGURE 3 Pathway analysis of discriminative metabolic alterations in experimental fibrosis with and without pirfenidone (PFD) treatment. Metabolic pathways are represented as circles according to their scores from enrichment (vertical axis) and topology analyses (pathway impact, horizontal axis) using MetaboAnalyst. a) Discriminative pathways in bleomycin-induced fibrosis compared with PBS controls. b) Discriminative pathways in bleomycin-induced fibrosis upon PFD treatment. c) Discriminative pathways in bleomycin-induced fibrosis upon PFD treatment compared with the PBS+PFD-treated group. d) Haematoxylin and eosin sections of lungs treated with PBS, PBS+PFD, bleomycin and B (bleomycin)+PFD are shown in the middle. Scale bar: $1000 \mu \mathrm{m}$. On the right, from top to bottom, representative images of metabolites reduced in fibrosis: phosphoenol pyruvate $(\mathrm{m} / \mathrm{z}$ 166.9750), bisphosphoglycerate $(\mathrm{m} / \mathrm{z}$ 264.9525), phosphatidic acid (m/z 647.4675) and phosphatidylethanolamine $(\mathrm{m} / \mathrm{z}$ 746.5140). On the left, from top to bottom, representative images of metabolites enhanced in fibrosis: ascorbic acid $(\mathrm{m} / \mathrm{z}$ 175.0250), glucose 6-phosphate $(\mathrm{m} / \mathrm{z} 259.0230), N$-acetylglucosamine phosphate $(\mathrm{m} / \mathrm{z} 300.0495)$ and UDP- $N$-acetyl-D-galactosamine $(\mathrm{m} / \mathrm{z}$ 606.0755). e) Relative quantification of metabolites depicted in (d) in mice treated with PBS, PBS+PFD, bleomycin and B+PFD. One-way ANOVA was used for statistical analysis, with the Bonferroni post hoc test. ${ }^{*}: p \leqslant 0.05 ;{ }^{* *}: p \leqslant 0.01 ;{ }^{* *}: p \leqslant 0.001$, between PBS and treated groups.

redox-related metabolites) in control (PBS and PBS+pirfenidone treatment), bleomycin-treated and bleomycin+pirfenidone-treated mice. Metabolite identification was performed using LC-MS on tissue MS/MS or accurate mass matching with databases (4 ppm mass accuracy; supplementary figures S2 and S3). Levels of nine out of 25 metabolites were significantly different between fibrotic and control tissue. Of the nucleotide derivatives, UMP and UDP- $N$-acetylglucosamine were significantly increased in both fibrosis and pirfenidone-treated fibrosis when compared with controls (figure 4a). We also found that the prostaglandin pathway was highly regulated. Although prostaglandin B1-A1 was unchanged, prostaglandins E2, G2, G1 and E2 glycerylester were significantly decreased in bleomycin- and bleomycin+pirfenidone-treated mice compared with controls (figure $4 \mathrm{~b}$ ). Lastly, we analysed metabolites from glycolysis and the TCA cycle, and found that 1,3-bisphosphoglycerate was significantly decreased in lungs from bleomycin- and bleomycin +pirfenidone-treated mice compared with controls, whereas glucose 6-phosphate was increased in fibrotic and pirfenidone-treated fibrotic tissue (figure 4c).

\section{Pharmacometabolic effects of pirfenidone in IPF}

To determine whether this analysis is useful to identify metabolic footprints of fibrosis in human lungs, as well as the pharmacometabolic effect of pirfenidone in IPF, we performed pathway analysis of discriminative metabolites in human lungs from rejected donors, explanted lungs of treatment-naive IPF patients and IPF patients on pirfenidone treatment at time of transplantation. In a similar approach as described earlier, we initially compared donor with untreated IPF tissue, to identify metabolic pathways putatively modulated during fibrosis (figure 5, table 1 and supplementary table S2). In total, 825 molecules were significantly different between donor and fibrotic tissue, with 480 molecules enriched and 345 molecules reduced in IPF. We then examined metabolic effects of pirfenidone in end-stage IPF by comparing explanted tissue from untreated and pirfenidone-treated IPF. From this analysis, 173 discriminative $\mathrm{m} / \mathrm{z}$ species were identified (figure 5 and supplementary table S2).

Metabolites responsible for metabolic differentiation were then subjected to pathway enrichment analysis; regulated metabolites driving pathway significance are listed in supplementary table S2. We identified pentose and glucuronate interconversions as pathways with the highest impact in IPF. Additionally, the

TABLE 1 Modulated metabolite pathways in mice and humans during fibrosis and upon pirfenidone treatment

\section{Reduced in fibrosis}

Glycolysis/gluconeogenesis

Linoleic acid metabolism

Inositol phosphate metabolism

Starch and sucrose metabolism

No

No

No

Yes

Enhanced in fibrosis

Pentose and glucuronate interconversions

Ascorbate and aldarate metabolism

Amino sugar and nucleotide sugar metabolism

Pentose phosphate pathway

Fructose and mannose metabolism

Vitamin B6 metabolism

Glutathione metabolism

\section{Yes}

Yes

Yes

Yes

Yes

No

No

Reduced upon pirfenidone

Ascorbate and aldarate metabolism

Enhanced upon pirfenidone

Glycolysis/gluconeogenesis 

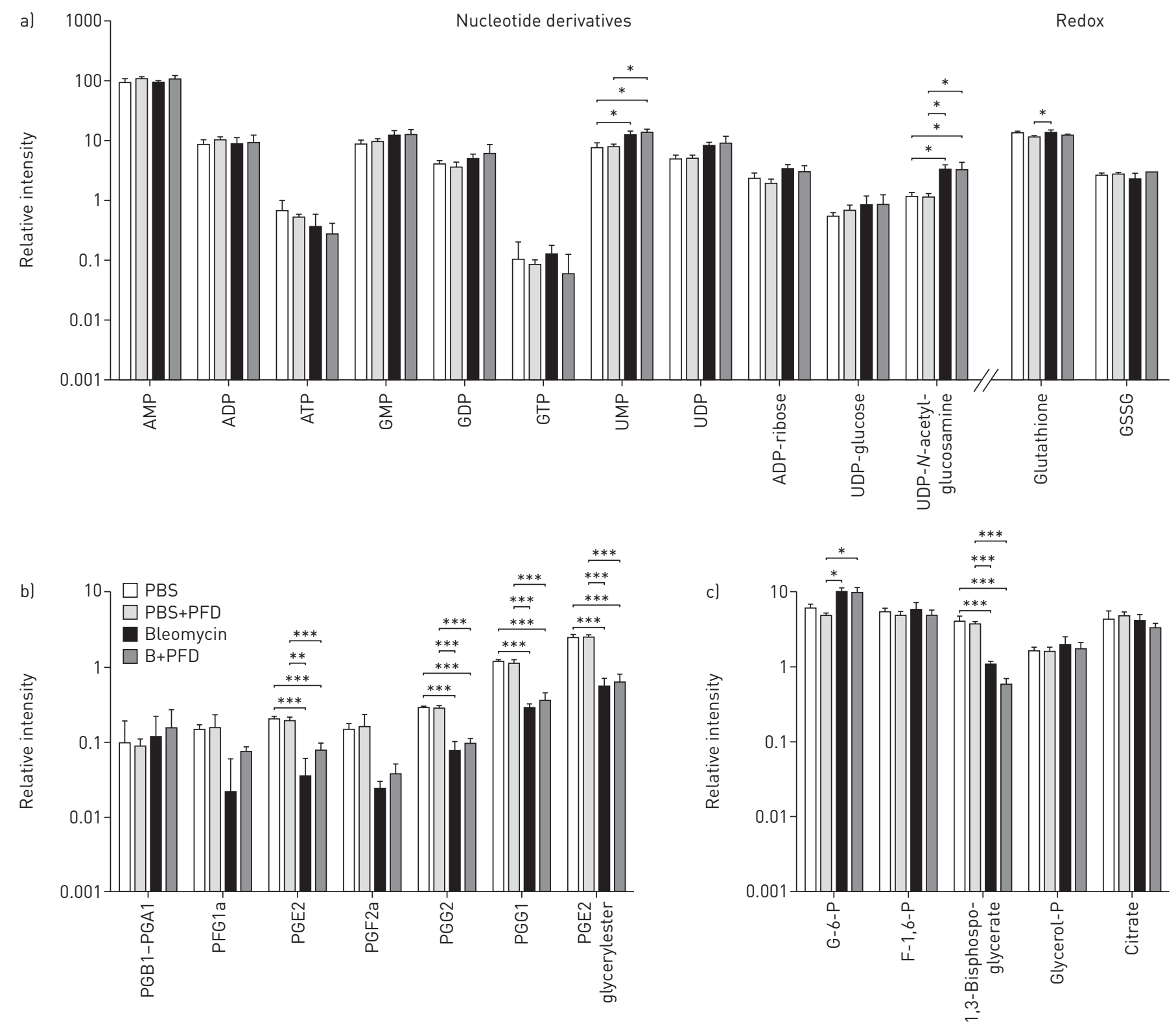

FIGURE 4 Relative abundance of endogenous tissue metabolites in mice treated with PBS, PBS+pirfenidone (PFD), bleomycin or bleomycin+PFD (B+PFD). Metabolites are grouped as a) nucleotide derivatives and redox-associated metabolites, b) prostaglandins, and $\mathrm{cl}$ glycolysis and the tricarboxylic acid cycle. Data are shown as mean $\pm S D(n=3)$ and represent the relative concentration of each group. One-way ANOVA was used for statistical analysis, with the Bonferroni post hoc test. GSSG: glutathione disulfide; PG: prostaglandin; G-6-P: glucose 6-phosphate; F-1,6-P: fructose 1,6-bisphosphate; glycerol-P: glycerol monophosphate. ${ }^{*}: p \leqslant 0.05 ;{ }^{* *}: p \leqslant 0.01 ;{ }^{* * *}: p \leqslant 0.001$, between PBS and treated groups.

ascorbate and aldarate pathways, as well as fructose and mannose metabolism, were upregulated in IPF, and vitamin B6 metabolism was increased in IPF compared with donor tissue (figure 5a, table 1 and supplementary table S2). Conversely, linoleic acid and inositol phosphate metabolism and the starch and sucrose pathways were downregulated in IPF. Comparison of explanted tissue from untreated and pirfenidone-treated IPF (figure 5b, table 1 and supplementary table S2) revealed signals from glycolysis/ gluconeogenesis were enhanced with pirfenidone. We then performed in situ localisation of key metabolites of the aforementioned pathways (figure $5 \mathrm{c}$ and $\mathrm{d}$ ), and confirmed both localisation and significant reduction of D-myo-inositol 1,4,5-trisphosphate and phosphatidylcholine in IPF and pirfenidone-treated IPF. In contrast, we observed localisation and a significant increase in 3-amino-2-oxopropyl phosphate and cysteinylglycine IPF and pirfenidone-treated IPF.

Lastly, we measured the relative abundance of 21 metabolites from several metabolic pathways (nucleotide derivatives, central pathway metabolites, prostaglandins and redox-related metabolites) in control, IPF and 
a)

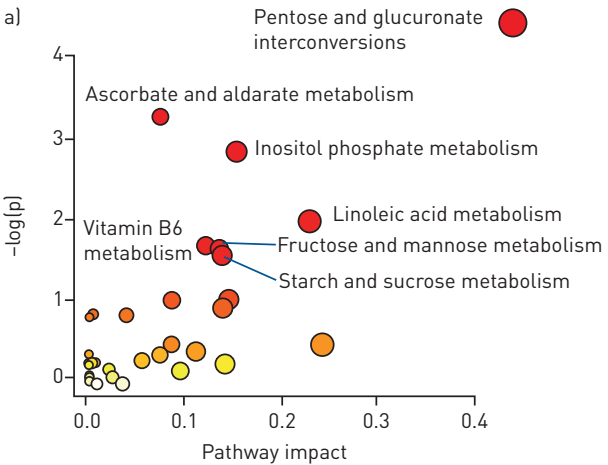

$\begin{array}{ll}\text { c) } & \begin{array}{c}\text { Reduced in IPF } \\ \text { Donor IPF+PFD }\end{array}\end{array}$

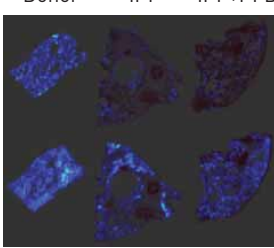

$\mathrm{MH}-(\mathrm{m} / \mathrm{z})$

418.9560

D-myo-inositol

1,4,5-trisphosphate

718.5410

Phosphatidylcholine

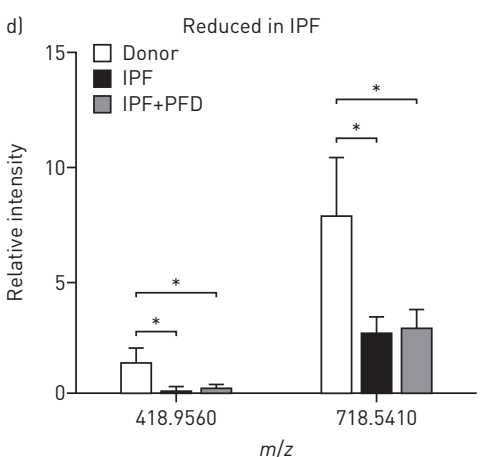

b)

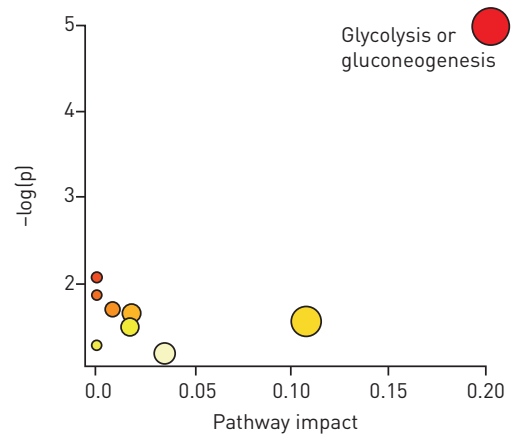

Enhanced in IPF

Donor IPF IPF+PFD
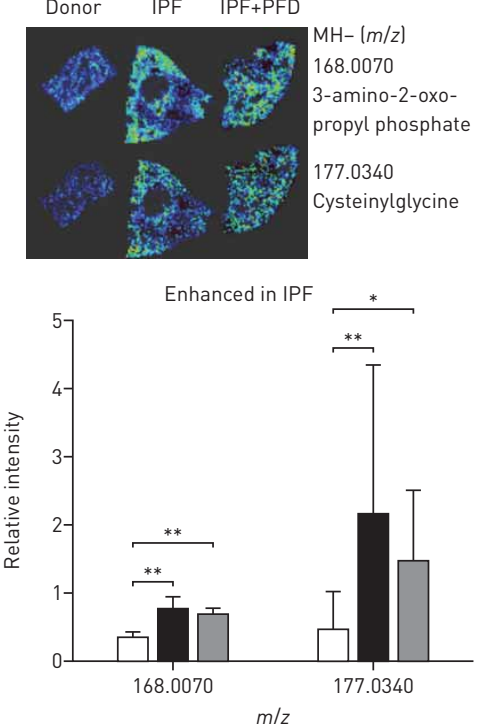

FIGURE 5 Pathway analysis of discriminative metabolic alterations in idiopathic pulmonary fibrosis (IPF) patients with and without pirfenidone (PFD) treatment. Metabolic pathways are represented as circles according to their scores from enrichment (vertical axis) and topology analyses (pathway impact, horizontal axis) using MetaboAnalyst. a) Discriminative pathways in IPF compared with donor. b) Discriminative pathways in IPF after PFD therapy. c) Haematoxylin and eosin sections of explanted lungs from donors, untreated IPF or PFD-treated IPF (IPF+PFD) patients are shown in the middle. Scale bar: $2000 \mu \mathrm{m}$. On the right, from top to bottom, representative images of metabolites reduced in IPF: D-myo-inositol 1,4,5-trisphosphate $1 \mathrm{~m} / \mathrm{z}$ 418.9560) and phosphatidylcholine $(\mathrm{m} / \mathrm{z}$ 718.5410). On the left, from top to bottom, representative images of metabolites enhanced in IPF: 3-amino-2-oxopropyl phosphate $(\mathrm{m} / \mathrm{z} 168.0070)$ and cysteinylglycine $(\mathrm{m} / \mathrm{z}$ 177.0340). d) Relative quantification of the metabolites depicted in (c) in donor, IPF and PFD-treated IPF (IPF+PFD). One-way ANOVA was used for statistical analysis, with the Bonferroni post hoc test. *: $\mathrm{p} \leqslant 0.05$; ${ }^{* *}: p \leqslant 0.01$, between donor and treated groups.

pirfenidone-treated IPF lungs; five out of 21 were significantly regulated. Of the nucleotide derivatives, ATP was significantly decreased in IPF and pirfenidone-treated IPF compared with control tissue (figure 6a). From the redox pathway, both glutathione and glutathione disulfide were significantly increased in pirfenidone-treated IPF compared with control tissue (figure 6a). The prostaglandin pathway was within the lower range of expression in humans, although prostaglandin G2 was significantly increased in IPF compared with controls. From glycolysis and the TCA cycle, glucose 6-phosphate was significantly increased in pirfenidone-treated IPF when compared with control tissue. Furthermore, fructose 1,6-bisphosphate, which is involved in glycolysis/gluconeogenesis, was significantly downregulated in IPF, suggesting deregulation of glycolysis/gluconeogenesis in this disease (figure 6c). Enhancement of the glycolysis/gluconeogenesis pathway upon pirfenidone administration (figure 5b) demonstrates a possible role for pirfenidone in restoring glycolysis in IPF.

\section{Discussion}

This proof-of-concept study demonstrated, for the first time, in situ metabolic alterations during fibrosis and the pharmacometabolic effect of pirfenidone on fibrotic tissue of mice and humans. Specifically, we found 

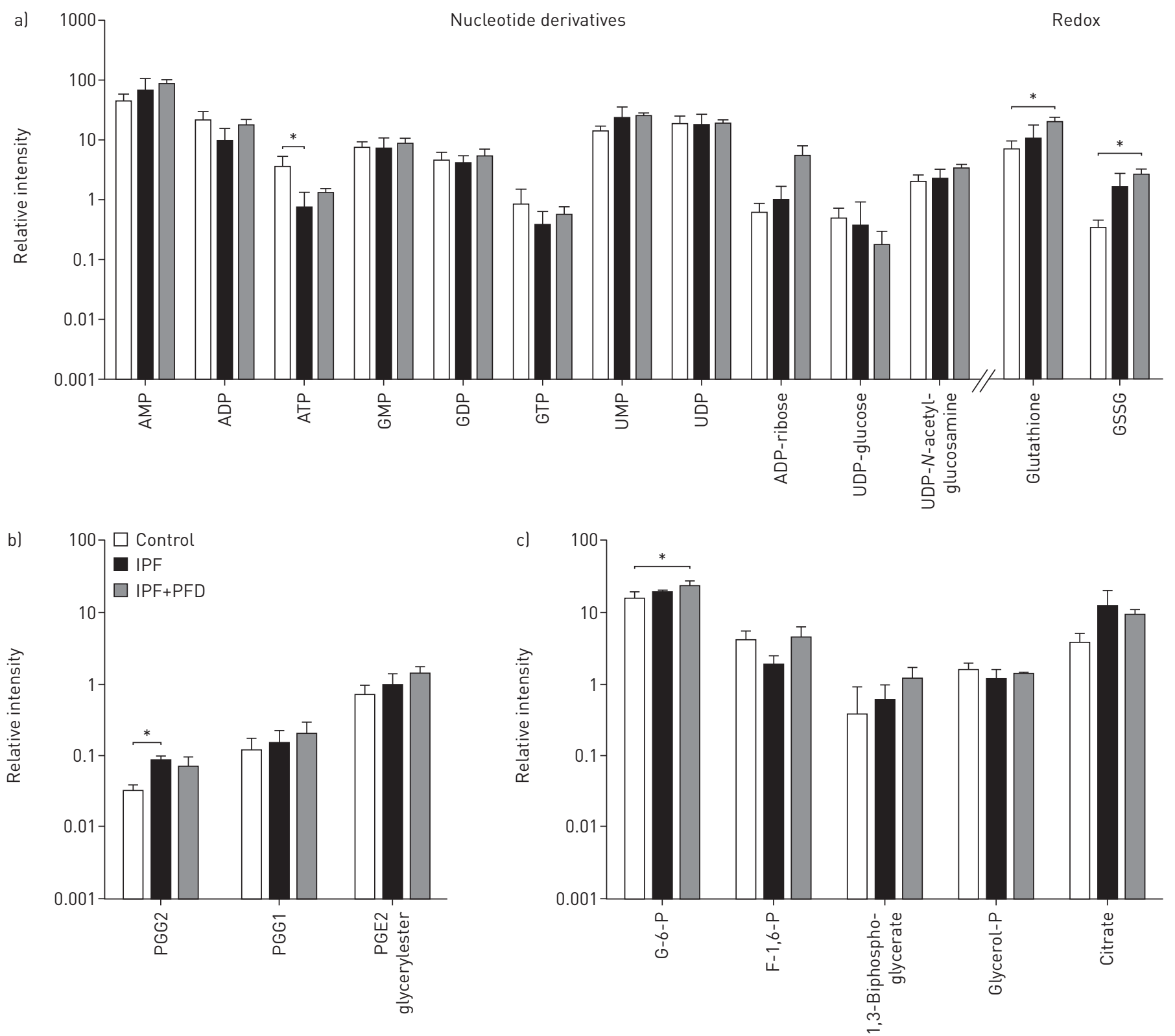

FIGURE 6 Relative abundance of endogenous tissue metabolites from donor, idiopathic pulmonary fibrosis (IPF) and pirfenidone (PFD)-treated IPF patients. Metabolites are grouped as a) nucleotide derivatives and redox-associated metabolites, b) prostaglandins, and cl glycolysis and the tricarboxylic acid cycle. Data are shown as mean $\pm S D(n=3)$ and represent the relative concentration of each group. One-way ANOVA was used for statistical analysis, with the Bonferroni post hoc test. GSSG: glutathione disulfide; PG: prostaglandin; G-6-P: glucose 6-phosphate; F-1,6-P: fructose 1,6-bisphosphate; glycerol-P: glycerol monophosphate. *: $p \leqslant 0.05$, between control and disease groups.

increased levels of pirfenidone and related metabolites in fibrotic tissue using high-resolution in situ MS. We detected mass spectra of fibrotic areas, to assess metabolite composition in specific regions of interest. Using this technology, we were then able to dissect metabolic pathways simultaneously in multiple samples and determine spatial distribution, as well as quantify intensity of detection. Lastly, we identified overlapping and exclusive metabolic fingerprints that putatively characterise fibrotic responses in humans and mice, together with pharmacometabolic responses to pirfenidone in fibrosis.

Due to the relatively small number of mice in each treatment group $(n=3)$, this should be viewed as a proof-of-concept study, exploring the utility of MALDI-FTICR-MSI for detection of metabolites in a mouse model of pulmonary fibrosis. To demonstrate statistical significance and reproducibility of our experiments, we performed hierarchical clustering and partial least squares discriminant analysis (supplementary figure S1). These data demonstrate clear separations between PBS-, bleomycin- and bleomycin+pirfenidone-treated groups, and underscore the power of this approach. 
Metabolomics is the study of global endogenous metabolite profiles generated by specific cellular processes. The presented metabolomic analyses has the potential to enhance understanding of fibrotic processes, promote discovery of diagnostic biomarkers, identify novel targets and improve current therapies. Critically, our data demonstrate that MALDI-FTICR-MSI can provide the microscopic and molecular analytic capabilities necessary for understanding the spatio-molecular features of complex microenvironments in IPF tissue.

Investigation of the distribution of pirfenidone and its related drug metabolites in the lung revealed 5-hydroxymethyl pirfenidone $(\mathrm{m} / z$ 202.0863) and 5-carboxy pirfenidone $(\mathrm{m} / z$ 216.0655) are globally more abundant in fibrotic lungs. However, in fibrotic lungs, they were abundantly localised in less-affected areas, with lower COL1 expression. This suggests the distribution and possibly the metabolism of pirfenidone are enhanced in fibrosis and accumulation in affected areas might be required for its antifibrotic effects. In lung fibroblasts, 5-hydroxymethyl pirfenidone and 5-carboxy pirfenidone inhibit transforming growth factor (TGF)- $\beta$-induced collagen synthesis, demonstrating these metabolites have antifibrotic activities [31].

Using ultra-sensitive MSI, we detected, and simultaneously visualised, thousands of endogenous metabolites in mouse and human lung sections, from diseased and control tissues. These include nucleotide derivatives, as well as central metabolic pathway metabolites, redox-related metabolites and prostaglandins, among others. We observed that intermediates in glycolysis, nucleotide metabolism and the pentose phosphate pathway are altered in fibrotic regions compared with healthy lungs. It was previously shown that glycolysis is altered in IPF [16]. KotTmann et al. [32] further suggested that dysregulated glycolysis results in elevated lactate levels, which may facilitate disease progression. This metabolic switch towards the oxidative pentose phosphate pathway in fibrosis might indicate the elevated energy demand and higher energy consumption present in fibrosis. Consistent with this, we found lower levels of ATP in IPF patients. Similarly, extracellular ATP is decreased in bronchoalveolar lavage fluid from IPF samples [33] and others have shown decreased intracellular ATP in IPF tissue [17], which may result from accumulation of dysfunctional mitochondria that promote fibrosis [34]. Collectively, these findings suggest an alternative energy cascade related to altered glycolysis in IPF.

Other metabolites identified in our dataset are related to redox mechanisms. Interestingly, we found unchanged levels of glutathione and glutathione disulfide in bleomycin-induced pulmonary fibrosis, whereas these are increased in pirfenidone-treated IPF patients. In mice, levels of glutathione and glutathione disulfide are suppressed by overexpression of TGF- $\beta$ [35]. We speculate that recovery of glutathione and glutathione disulfide levels in IPF might indicate a role for pirfenidone in modulating the redox imbalance that contributes to persistent fibrosis [36].

Remarkably, we observed increased ascorbate and aldarate metabolism in both mice and humans with fibrosis. This is consistent with the enhancement in collagen metabolism and production observed in organ fibrosis. In mice, ascorbate and aldarate metabolism decrease after pirfenidone treatment. Ascorbate is a crucial cosubstrate for enzymes catalysing post-translational hydroxylation of prolyl and lysyl residues necessary for thermostability of the collagen triple helix and extracellular cross-linking of collagen fibres [37, 38], making it critical for collagen synthesis and processing. Thus, enhancement of ascorbate and aldarate metabolism during fibrosis, and the corresponding decrease after pirfenidone administration, are indicative of its antifibrotic activity. These data are also consistent with the effect of pirfenidone on collagen processing and support its role in inhibiting collagen fibril formation in IPF fibroblasts [39].

Significant alteration of lipids in fibrotic regions is consistent with recent studies demonstrating that lipids, particularly phospholipids, are increased in fibrosis and can act as mediators in lipid signalling pathways [40-43]. Additionally, prostaglandins, which are suggested to have antifibrotic activities in lung injury [44], were also reduced in fibrotic regions, implying these may serve as indicators, and potentially relevant targets, for pulmonary fibrosis treatment.

Pathway enrichment analysis further revealed putative overlapping, as well as, distinctive features in mouse and human fibrosis (figure 3, table 1, and supplementary tables S1 and S2), and several factors may account for these differences. First, we note bleomycin-induced pulmonary fibrosis is a self-resolving, easily manipulated model, which does not fully recapitulate human IPF, although it does show strong commonalities on distinct levels, supporting continuous use for pre-clinical testing [45]. Second, pirfenidone treatment in mice occurred from disease initiation to peak of fibrosis, whereas our IPF cohort was at end-stage disease, requiring transplantation. Thus, our data may also reflect important differences in treatment responses at different disease stages.

IPF is a multifactorial and complex disease, and bleomycin-induced fibrosis remains the most studied model of lung fibrosis. Kinetic analyses to study the metabolic effects of pirfenidone treatment and metabolic pathway modifications occurring early after pirfenidone administration are therefore limited to 
this model. For example, pirfenidone and its metabolites are maximally detectable in lungs 45 min post-administration [18]. Thus, we successfully detected increased levels of pirfenidone and its related metabolites in mouse, but not human, fibrotic lungs. This may result from timely, controlled drug administration in mice, which is difficult to recapitulate clinically in pre-transplanted patients immediately preceding surgical procedures. Despite the aforementioned factors, with the introduction of transbronchial cryobiopsies for earlier diagnosis of diffuse parenchymal lung diseases [46], differences can be minimised and the applicability of MALDI imaging for metabolite profiling or pharmacometabolic drug responses should be considered.

Collectively, our findings suggest metabolic profiles are altered in regions affected by pulmonary fibrosis. Although results are preliminary, based on this proof-of-concept study we propose that the combination of pharmaco- and metabolomics utilised in this study may ultimately lead to improved understanding of both the disease mechanisms of IPF and the mechanism of action of pirfenidone. Moreover, elucidating pirfenidone-associated metabolism and its cellular mechanism of action may facilitate prediction of individual responses to antifibrotic drugs. Label-free MALDI-MSI techniques allow simultaneous detection, visualisation and quantification of both exogenous (drugs and related metabolites) and endogenous metabolites, providing competitive advantages over traditional analytical techniques. Thus, we anticipate MALDI-MSI techniques for determining spatiotemporal behaviours of drugs and endogenous metabolites will be broadly applicable to drug efficacy studies and elucidating the metabolism of bioactive small molecules involved in fibrosis and other pathological conditions.

Acknowledgements: The authors would like to thank Claudia-Marieke Pflüger, Ulrike Buchholz, Gabriele Mettenleiter and Andreas Voss from the Research Unit Analytical Pathology (Helmholtz Zentrum München, Neuherberg, Germany), as well as Constanze Heise and Daniela Dietel from the Comprehensive Pneumology Center (Helmholtz Zentrum München, Munich, Germany), for providing technical assistance.

Author contributions: A. Walch, O. Eickelberg, N. Sun and I.E. Fernandez designed the study. N. Sun, I.E. Fernandez, M. Wei and M. Witting performed the experiments. S.E. Verleden provided lung tissues from healthy donors, IPF and pirfenidone-treated IPF lung tissue. N. Sun and I.E. Fernandez drafted the manuscript. I.E. Fernandez, N. Sun, A. Walch, O. Eickelberg and M. Aichler analysed and interpreted the results. I.E. Fernandez, N. Sun, A. Walch, O. Eickelberg, S.E. Verleden, M. Aichler, M. Wei, M. Witting and P. Schmitt-Kopplin drafted and edited the manuscript for important intellectual content. G. Burgstaller and A. Feuchtinger supported immunofluorescence experiments.

Conflict of interest: None declared.

Support statement: The study was funded by Helmholtz Zentrum München (TKP-Project), the German Center for Lung Research (DZL), the Ministry of Education and Research of the Federal Republic of Germany (BMBF; 01ZX1310B and 01KT1615), and the Deutsche Forschungsgemeinschaft (HO 1254/3-1, SFB 824 C4, CRC/Transregio 205/1 "The Adrenal: Central Relay in Health and Disease") to A. Walch. Funding information for this article has been deposited with the Crossref Funder Registry.

\section{References}

1 Fernandez IE, Eickelberg O. New cellular and molecular mechanisms of lung injury and fibrosis in idiopathic pulmonary fibrosis. Lancet 2012; 380: 680-688.

2 Schaefer CJ, Ruhrmund DW, Pan L, et al. Antifibrotic activities of pirfenidone in animal models. Eur Respir Rev 2011; 20: 85-97.

3 Lasky J. Pirfenidone. IDrugs 2004; 7: 166-172.

4 Raghu G, Collard HR, Egan JJ, et al. An official ATS/ERS/JRS/ALAT statement: idiopathic pulmonary fibrosis: evidence-based guidelines for diagnosis and management. Am J Respir Crit Care Med 2011; 183: 788-824.

$5 \quad$ Carter NJ. Pirfenidone: in idiopathic pulmonary fibrosis. Drugs 2011; 71: 1721-1732.

6 Kakugawa T, Mukae H, Hayashi T, et al. Pirfenidone attenuates expression of HSP47 in murine bleomycin-induced pulmonary fibrosis. Eur Respir J 2004; 24: 57-65.

7 Cornett DS, Frappier SL, Caprioli RM. MALDI-FTICR imaging mass spectrometry of drugs and metabolites in tissue. Anal Chem 2008; 80: 5648-5653.

8 Römpp A, Guenther S, Takats Z, et al. Mass spectrometry imaging with high resolution in mass and space $\left(\mathrm{HR}^{2}\right.$ MSI) for reliable investigation of drug compound distributions on the cellular level. Anal Bioanal Chem 2011; 401: 65-73.

9 Castellino S, Groseclose MR, Wagner D. MALDI imaging mass spectrometry: bridging biology and chemistry in drug development. Bioanalysis 2011; 3: 2427-2441.

10 Kim YH, Fujimura Y, Hagihara T, et al. In situ label-free imaging for visualizing the biotransformation of a bioactive polyphenol. Sci Rep 2013; 3: 2805.

11 Miura D, Fujimura Y, Wariishi $\mathrm{H}$. In situ metabolomic mass spectrometry imaging: recent advances and difficulties. J Proteomics 2012; 75: 5052-5060.

12 Balluff $\mathrm{B}$, Schone $\mathrm{C}$, Hofler $\mathrm{H}$, et al. MALDI imaging mass spectrometry for direct tissue analysis: technological advancements and recent applications. Histochem Cell Biol 2011; 136: 227-244.

13 Abrosimov LI, Karasik VE, Beliakova IP. [Hygienic principles of physical load standardizing in physical education of school children.] Vestn Ross Akad Med Nauk 1993: 13-19.

14 Walch A, Rauser S, Deininger SO, et al. MALDI imaging mass spectrometry for direct tissue analysis: a new frontier for molecular histology. Histochem Cell Biol 2008; 130: 421-434. 
15 Aichler M, Borgmann D, Krumsiek J, et al. $N$-acyl taurines and acylcarnitines cause an imbalance in insulin synthesis and secretion provoking beta cell dysfunction in type 2 diabetes. Cell Metab 2017; 25: 1334-1347.

16 Zhao YD, Yin L, Archer S, et al. Metabolic heterogeneity of idiopathic pulmonary fibrosis: a metabolomic study. BMJ Open Respir Res 2017; 4: e000183.

17 Kang YP, Lee SB, Lee JM, et al. Metabolic profiling regarding pathogenesis of idiopathic pulmonary fibrosis. J Proteome Res 2016; 15: 1717-1724.

18 Sun N, Fernandez IE, Wei M, et al. Pharmacokinetic and pharmacometabolomic study of pirfenidone in normal mice tissues using high mass resolution MALDI-FTICR-mass spectrometry imaging. Histochem Cell Biol 2016; 145: 201-211.

19 Fernandez IE, Amarie OV, Mutze K, et al. Systematic phenotyping and correlation of biomarkers with lung function and histology in lung fibrosis. Am J Physiol Lung Cell Mol Physiol 2016; 310: L919-L927.

20 Verleden SE, Vasilescu DM, Willems S, et al. The site and nature of airway obstruction after lung transplantation. Am J Respir Crit Care Med 2014; 189: 292-300.

21 Boon M, Verleden SE, Bosch B, et al. Morphometric analysis of explant lungs in cystic fibrosis. Am J Respir Crit Care Med 2016; 193: 516-526.

22 Sun N, Ly A, Meding S, et al. High-resolution metabolite imaging of light and dark treated retina using MALDI-FTICR mass spectrometry. Proteomics 2014; 14: 913-923.

23 Buck A, Ly A, Balluff B, et al. High-resolution MALDI-FT-ICR MS imaging for the analysis of metabolites from formalin-fixed paraffin-embedded clinical tissue samples. J Pathol 2015; 237: 123-132.

24 Palmer A, Phapale P, Chernyavsky I, et al. FDR-controlled metabolite annotation for high-resolution imaging mass spectrometry. Nat Methods 2017; 14: 57-60.

25 Wishart DS, Tzur D, Knox C, et al. HMDB: the Human Metabolome Database. Nucleic Acids Res 2007; 35: D521-D526.

26 Xia J, Wishart DS. MetPA: a web-based metabolomics tool for pathway analysis and visualization. Bioinformatics 2010; 26: 2342-2344

27 Alexandrov T, Becker M, Deininger SO, et al. Spatial segmentation of imaging mass spectrometry data with edge-preserving image denoising and clustering. J Proteome Res 2010; 9: 6535-6546.

28 Alexandrov T, Becker M, Guntinas-Lichius O, et al. MALDI-imaging segmentation is a powerful tool for spatial functional proteomic analysis of human larynx carcinoma. J Cancer Res Clin Oncol 2013; 139: 85-95.

29 Albeiroti S, Soroosh A, de la Motte CA. Hyaluronan's role in fibrosis: a pathogenic factor or a passive player? Biomed Res Int 2015; 2015: 790203.

30 Li Y, Jiang D, Liang J, et al. Severe lung fibrosis requires an invasive fibroblast phenotype regulated by hyaluronan and CD44. I Exp Med 2011; 208: 1459-1471.

31 Togami K, Kanehira Y, Tada H. Possible involvement of pirfenidone metabolites in the antifibrotic action of a therapy for idiopathic pulmonary fibrosis. Biol Pharm Bull 2013; 36: 1525-1527.

32 Kottmann RM, Kulkarni AA, Smolnycki KA, et al. Lactic acid is elevated in idiopathic pulmonary fibrosis and induces myofibroblast differentiation via $\mathrm{pH}$-dependent activation of transforming growth factor-beta. Am J Respir Crit Care Med 2012; 186: 740-751.

33 Riteau N, Gasse P, Fauconnier L, et al. Extracellular ATP is a danger signal activating P2X7 receptor in lung inflammation and fibrosis. Am J Respir Crit Care Med 2010; 182: 774-783.

34 Bueno M, Lai YC, Romero Y, et al. PINK1 deficiency impairs mitochondrial homeostasis and promotes lung fibrosis. J Clin Invest 2015; 125: 521-538.

35 Lu HI, Huang TH, Sung PH, et al. Administration of antioxidant peptide SS-31 attenuates transverse aortic constriction-induced pulmonary arterial hypertension in mice. Acta Pharmacol Sin 2016; 37: 589-603.

36 Kurundkar A, Thannickal VJ. Redox mechanisms in age-related lung fibrosis. Redox Biol 2016; 9: 67-76.

37 Hata R, Senoo H. L-ascorbic acid 2-phosphate stimulates collagen accumulation, cell proliferation, and formation of a three-dimensional tissuelike substance by skin fibroblasts. J Cell Physiol 1989; 138: 8-16.

38 Murad S, Tajima S, Johnson GR, et al. Collagen synthesis in cultured human skin fibroblasts: effect of ascorbic acid and its analogs. J Invest Dermatol 1983; 81: 158-162.

39 Knuppel L, Ishikawa Y, Aichler M, et al. A novel antifibrotic mechanism of nintedanib and pirfenidone. Inhibition of collagen fibril assembly. Am J Respir Cell Mol Biol 2017; 57: 77-90.

40 Castelino FV. Lipids and eicosanoids in fibrosis: emerging targets for therapy. Curr Opin Rheumatol 2012; 24: 649-655.

41 Mouratis MA, Aidinis V. Modeling pulmonary fibrosis with bleomycin. Curr Opin Pulm Med 2011; 17: 355-361.

42 Tager AM, LaCamera P, Shea BS, et al. The lysophosphatidic acid receptor LPA1 links pulmonary fibrosis to lung injury by mediating fibroblast recruitment and vascular leak. Nat Med 2008; 14: 45-54.

43 Patel RB, Kotha SR, Sherwani SI, et al. Pulmonary fibrosis inducer, bleomycin, causes redox-sensitive activation of phospholipase D and cytotoxicity through formation of bioactive lipid signal mediator, phosphatidic acid, in lung microvascular endothelial cells. Int J Toxicol 2011; 30: 69-90.

44 Huang SK, Peters-Golden M. Eicosanoid lipid mediators in fibrotic lung diseases: ready for prime time? Chest 2008; 133: 1442-1450.

45 Jenkins RG, Moore BB, Chambers RC, et al. An Official American Thoracic Society Workshop Report: Use of Animal Models for the Preclinical Assessment of Potential Therapies for Pulmonary Fibrosis. Am J Respir Cell Mol Biol 2017; 56: 667-679.

46 Hetzel J, Maldonado F, Ravaglia C, et al. Transbronchial cryobiopsies for the diagnosis of diffuse parenchymal lung diseases: expert statement from the Cryobiopsy Working Group on Safety and Utility and a call for standardization of the procedure. Respiration 2018; 95: 188-200. 